\title{
Establishment and characterization of a novel cell line derived from human thymoma $A B$ tumor
}

\author{
Yesim Gökmen-Polar ${ }^{1}$, Kerry L Sanders ${ }^{1}$, Chirayu P Goswami ${ }^{2}$, Oscar D Cano ${ }^{1}$, Narjis A Zaheer ${ }^{3}$, Rohit K Jain ${ }^{3}$, \\ Kenneth A Kesler ${ }^{4}$, Robert P Nelson $\mathrm{Jr}^{1}$, Gail H Vance ${ }^{5}$, Danielle Smith ${ }^{5}$, Lang Li ${ }^{2}$, Angelo A Cardoso ${ }^{1}$, \\ Sunil Badve ${ }^{1,3}$, Patrick J Loehrer $\mathrm{Sr}^{1}$ and George W Sledge $\mathrm{Jr}^{1,3}$
}

Thymomas are low-grade epithelial tumors of the anterior mediastinum. The complexity of the disease and the lack of in vitro and in vivo models hamper the development of better therapeutics. In this study, we report a novel cell line, designated as IU-TAB-1, which was established from a patient with stage II thymoma (World Health Organization-type AB). The IU-TAB-1 cell line was established in vitro and characterized using histological and immunohistochemical staining, fluorescence-activated cell sorting, cytogenetic analyses and functional assays including in vitro and a NOD/SCID xenograft model. A whole-genome gene expression analysis (Illumina) was performed on the IU-TAB-1 cell line and 34 thymomas to determine the clinical relevance of the cell line. The IU-TAB-1 cell line was positive for epithelial markers (pan-cytokeratin and EpCAM/CD326) including thymic epithelial (TE) surface markers (such as CD29, CD9, CD54/ICAM-1, CD58 and CD24) and p63, and negative for B- and T-cell lineage markers. Gene expression profiling demonstrated overlapping and distinct genes between IU-TAB-1 and primary thymomas including the primary tumor (from which the cell line was derived). IUTAB-1 cells are tumorigenic when implanted in immunodeficient mice with tumors reaching a volume of $1000 \mathrm{~mm}^{3}$ at around 130 days. The established cell line represents a biologically relevant new tool to investigate the molecular pathology of thymic malignancies and to evaluate the efficacy of novel therapeutics both in vitro and in vivo.

Laboratory Investigation (2012) 92, 1564-1573; doi:10.1038/labinvest.2012.115; published online 27 August 2012

KEYWORDS: gene expression analysis; human epithelial cancer cell lines; thymoma

Thymomas are rare epithelial tumors of the thymus gland with diverse pathological findings and clinical outcomes. The classification of these tumors is controversial and the commonly used World Health Organization (WHO) unifying schema categorizes thymic tumors into thymomas (subtypes $\mathrm{A}, \mathrm{AB}$ and $\mathrm{B} 1-\mathrm{B} 3$ ) and thymic carcinomas (formerly grouped as type C). ${ }^{1,2}$ The current therapeutic management is almost entirely based on tumor stage. ${ }^{3}$ Although complete resection and chemotherapy are effective therapies for early disease, metastases remain a challenge. ${ }^{4-6}$ Tumors of all histological types, albeit with different frequencies, can spread locally within the pleura and pericardial cavity, and give rise to local or distant metastases. ${ }^{5,6}$ The treatment strategies include the combination of surgery, radiation and chemotherapy based on the extent of the disease and the status of tumor resectability. ${ }^{3,4}$ Surgery is the main choice of treatment for localized thymic tumors, as complete resection is the most significant prognostic factor. In patients with advanced thymic malignancies, cisplatin and carboplatin-based combination chemotherapy has become standard. ${ }^{7,8}$ Though remissions may be of significant duration, chemotherapy alone is not felt curative with relapses in unresectable patients being almost universal. Patients with locally advanced and metastatic thymoma have a reduced disease-free survival. Second cancers and associated immunological disorders also contribute to decreased overall survival of these patients. ${ }^{9}$

Given the rarity of the thymic epithelial (TE) neoplasms, new treatments are based on the studies extrapolated from other epithelial tumors rather than biology-driven therapeutic strategies. No mechanistic studies exist to predict the unique predilection for pleural disease recurrence and

\footnotetext{
'Department of Medicine, Indiana University School of Medicine, Indianapolis, IN, USA; ${ }^{2}$ Center for Computational Biology and Bioinformatics, Indiana University School of Medicine, Indianapolis, IN, USA; ${ }^{3}$ Department of Pathology and Laboratory Medicine, Indiana University School of Medicine, Indianapolis, IN, USA; ${ }^{4}$ Department of Surgery, Indiana University School of Medicine, Indianapolis, IN, USA and ${ }^{5}$ Department of Medical and Molecular Genetics, Indiana University School of Medicine, Indianapolis, IN, USA

Correspondence: Dr Y Gökmen-Polar, PhD, Department of Medicine, Indiana University School of Medicine, Walther Hall, 980W Walnut Street, C230, IN, IN 46202, USA. E-mail: ypolar@iupui.edu
} 
metastasis, or the development of second malignancies. Recent comprehensive genomic analysis has shown molecular distinctions between different histological types of thymic tumors. ${ }^{10}$ Although targeted therapies directed against epidermal growth factor receptor (EGFR) and KIT are available, ${ }^{11,12}$ but these, unlike in lung cancer, do not appear to be efficacious (PJL, unpublished data). Preclinical evaluation of efficacy of novel therapies is limited due to the lack of representative cellular and animal models. Among the four cell lines reported to date, three were isolated from thymic carcinoma patients, ${ }^{13-15}$ while one cell line was established from type B1 thymoma. ${ }^{14}$ To the best of our knowledge, this is the first human spindle cell thymoma cell line derived from a patient with type ' $\mathrm{AB}$ ' thymoma. We further explored its clinical relevance in comparison with the primary thymic tumors, and our studies support its usefulness as a preclinical tool in TE malignancies.

\section{MATERIALS AND METHODS}

\section{Brief Clinical Summary of the Patient}

A 53-year-old male with 35 pack-year history of smoking presented with an upper respiratory infection. Chest radiograph revealed a $6.5-\mathrm{cm}$ anterior mediastinal mass. He did not have any history of radiation exposure or symptoms of paraneoplastic syndromes, including myasthenia gravis. At operation, the tumor was found to be adherent to the lung and pericardium hence an en bloc thymectomy with partial pericardectomy and wedge resection of the right upper lobe was performed. Pathology revealed a thymoma of WHO AB type with microscopically invasion of adjacent adipose tissue. The tumor, in all the 13 sections examined, was composed of spindle cells admixed with variable number of lymphocytes (B-like areas). A separate type B-cell component was not identified. All resection margins, peritumoral lymph nodes and the adherent lung were negative for tumor (stage II thymoma (T2N0M0)). Adjuvant radiotherapy was considered but not given and the patient was disease-free at the time of last follow-up. As the patient did not have signs or symptoms of immune-mediated illness or recurrent infections a detailed immunological evaluation was not performed.

\section{Establishment of the Cell Line}

An IRB-approved written informed consent was obtained from the patient to conduct research with his resected tumor. A piece of sterile tumor sample from this patient was used to establish the cell line. The tumor was cut into small pieces in a sterile $100-\mathrm{mm}$ tissue plastic dish. Tissues were cultured in RPMI-1640 medium (Mediatec, Manassas, VA, USA) supplemented with $5 \% \mathrm{FBS}, 1 \%$ penicillin and streptomycin in a $5 \% \mathrm{CO}_{2}$ atmosphere at $37^{\circ} \mathrm{C}$. The medium was changed every 4 days. To remove fibroblasts, we applied a number of methods including keeping the serum concentration at 5\% FBS, selective trypsinization and adhesion techniques as described by Langdon. ${ }^{16}$ After 2 weeks, cells were transferred into $75-\mathrm{cm}^{2}$ flasks. The flasks were regularly assessed for epithelial cells outgrowth. The pure population of epithelial cells was designated as IU-TAB-1.

\section{Immunohistochemistry of the Primary Tumor and the Established Thymoma Cell Line, IU-TAB-1}

Cells were examined by light microscope using phase contrast and methylene blue staining according to the manufacturer's instructions (Fisher Scientific, Pittsburgh, PA, USA). Cytological preparations and cell blocks were made for the cells and a comparative analysis was performed with the primary tumor using hematoxylin and eosin (H\&E) morphology and a number of immunohistochemical stains (CD1a, CD20, CD99, TdT, p63, EGFR and c-KIT). The antibodies were purchased from Dako (Carpinteria, CA, USA) and immunohistochemistry was performed according to the manufacturer's instructions.

\section{Immunophenotype by Flow Cytometric Analysis}

Expression of surface markers was assessed using fluorochrome-conjugated-specific antibodies followed by flow cytometry analysis. The following antibodies were used: FITC-conjugated CD3, CD8, CD9, CD10, CD13, CD15, CD16, CD18, CD19, CD21, CD34, CD40L/CD154, CD45, CD49b, CD49d, CD49e, CD54/ICAM-1, CD90 and CD100; PE-conjugated CD4, CD6, CD24, CD29, CD38, CD40, CD49a, CD49c, CD49f, CD50, CD51/CD61 ( $\alpha \mathrm{v} \beta 3)$ CD58, CD62L, CD95, CD106, CD117, CD133, CD134, CD138, CD140a/PDGFR $\alpha$, CD140b/PDGFR $\beta$, CD142, CD146, EpCAM/CD326, NG2, EGFR and FLT3. Antibodies were obtained from BD Biosciences (San Jose, CA, USA), BD/ PharMingen (San Diego, CA, USA) or Beckman Coulter (Miami, FL, USA). Cells were labeled for $30 \mathrm{~min}$ at $4{ }^{\circ} \mathrm{C}$; irrelevant isotype-matched antibodies were used as negative controls. Samples were acquired in a BD FACSCalibur EPC flow cytometer, and at least 10000 -gated events were acquired for each sample.

\section{Chromosome Analyses}

G-banded karyotyping was performed on IU-TAB-1 cell line (passage 10). Dividing cells were prepared for conventional cytogenetic analysis following standard protocols. Cells were dropped onto cold, wet glass slides and then aged at $90^{\circ} \mathrm{C}$ for 60 and $20 \mathrm{~min}$ at $65^{\circ} \mathrm{C}$ followed by GTG banding. In total, 20 dividing cells were analyzed. Karyograms were prepared with an imaging system (Genetix, Santa Clara, CA, USA).

\section{Microarray Analysis}

RNA isolation and whole-genome-cDNA-mediated annealing, selection, extension and ligation (WGDASL; Illumina, San Diego, CA, USA) of IU-TAB-1 (quadruplets for the cell line) and 34 thymomas were performed according the manufacturer's instructions. The probe set used was the Illumina Human Ref-8BeadChip. This BeadChip features up to date content covering $>24000$ annotated genes derived 
Table 1 Chromosome aberrations detected in IU-TAB-1 cell line in comparison with other histological types

\begin{tabular}{|c|c|c|}
\hline Genetic aberrations & Histological types reported & IU-TAB-1 \\
\hline \multirow[t]{2}{*}{ 1q gain } & B2-B3 and thymic carcinoma; ${ }^{10,23,24}$ & Not identified \\
\hline & $A, A B^{21,22}$ & \\
\hline $1 q-1 q 21$ and $1 p$ & $A B$ and all types $10,21,22$ & Present \\
\hline 2 & $A B$ and all types 22,23 & Present \\
\hline 3 & $\begin{array}{l}A B \text { and } B 2-B 3 \text { and thymic carcino- } \\
\text { mas }^{10,21-23,24}\end{array}$ & Present \\
\hline $4 p$ gain & $\mathrm{B} 3$ and thymic carcinoma ${ }^{10}$ & Not identified \\
\hline 4 & $A B$ and all types 22,23 & Present \\
\hline 5 & $A B$ and all types $21-23$ & Present \\
\hline $5 q 21-22$ & $B 2$ and invasive $B 3^{21,24}$ & Not identified \\
\hline $6 p 21.3$ & All types ${ }^{21-24}$ & Not identified \\
\hline$r(6)$ & $A B^{33}$ & Not identified \\
\hline $6 q 23-q 25$ & $A B$ and all types $10,21,23$ & Present \\
\hline \multirow[t]{2}{*}{$7 p 15$} & B2, B3, thymic carcinoma and some & Present \\
\hline & AB thymomas ${ }^{21}$ & \\
\hline $8 p 11.21$ & B2, B3, thymic carcinoma ${ }^{21}$ & Not identified \\
\hline $8 p 21$ & AB and $B$ types 21,23 & Present \\
\hline $8 q 11$ & $\mathrm{~B} 2$ and $\mathrm{B} 3$ & Not identified \\
\hline 9 & $A, A B, B 1^{22,23}$ & Not identified \\
\hline $9 p$ loss & A, thymic carcinoma ${ }^{22}$ & Not identified \\
\hline $10 p$ & $A B^{21}$ & Present \\
\hline $11 q 21-23$ & $A B^{21}$ & Present \\
\hline $12 p$ & $\mathrm{~B} 2, \mathrm{~B}^{22}$ & Not identified \\
\hline $13 q$ & $A B$ and all types ${ }^{10,21-23}$ & Present \\
\hline \multirow[t]{2}{*}{$13 q 14.3$} & B2, B3, thymic carcinoma and some & Not identified \\
\hline & $A B$ thymomas ${ }^{21}$ & \\
\hline $16 p$ gain & $\mathrm{A}, \mathrm{B} 2^{22}$ & Not identified \\
\hline $16 q$ & AB and all types $21,22,24$ & Present \\
\hline $16 q 22.1$ & B2, invasive B3, thymic carcinoma ${ }^{21,24}$ & Not identified \\
\hline$(16 ; 12)(q 11 ; p 11.2)$ & $A B^{34}$ & Not identified \\
\hline 17p gain & $A, A B^{22}$ & Not identified \\
\hline \multirow[t]{2}{*}{$17 p 13$ loss } & Invasive B3 and thymic & Not identified \\
\hline & carcinoma $10,21,22,24$ & \\
\hline \multirow[t]{2}{*}{ 17q gain } & B3 and thymic carcinoma and some & Not identified \\
\hline & $A B^{10,22,24}$ & \\
\hline $17 q$ & $A B^{21,22}$ & Present \\
\hline 18q gain & B3 and thymic carcinoma $a^{10,24}$ & Not identified \\
\hline $18 q$ & AB and B types 22,23 & Present \\
\hline 20 & $\mathrm{~A}, \mathrm{~B} 2, \mathrm{~B} 322$ & Present \\
\hline 22 & $A^{22}$ & Present \\
\hline
\end{tabular}

from RefSeq (build 36.2, release 22). The clinical details of the patients were described in Table $1 .{ }^{17}$ The tumors were categorized into three groups: group I $(n=9)$-type $\mathrm{AB}$; group
II ( $n=19)$-types B1-2; and group III $(n=6)$-type B3. Briefly, data on 34 patients from Illumina Human WGDASL arrays, with each array containing 18401 probes were analyzed. Genes that had a poor signal quality across a maximal number of arrays were filtered out. As a result, 8260 genes were found to have signals significantly above background. The samples were run in three batches and batch effect was removed statistically using Partek Genomics suite's batch effect removal tool. The data were quantile normalized and $\log _{2}$ transformed before statistical analysis. After quantile normalization, unsupervised hierarchical clustering was performed. In addition, comparisons were made between cell line, original tumor sample and other primary tumors for TE cell surface antigens, ${ }^{18}$ stromal gene signatures ${ }^{19}$ and immune-system-related genes. ${ }^{20}$

\section{In vitro Growth Properties of IU-TAB-1 Cell Line}

Cell growth was assessed both by trypan-blue dye (Sigma, St Louis, MO, USA) exclusion and bromodeoxyuridine (BrdU) cell proliferation method (Roche Diagnostics, Indianapolis, IN, USA). IU-TAB- 1 cells were plated as $0.5 \times 10^{6}$ cells in a $60-\mathrm{mm}$ plate (trypan blue) or 8000 cells/well in a 96-well plate, and allowed to attach overnight. Cell viability (trypan blue dye exclusion) and cell proliferation (BrdU cell colorimetric ELISA kit) were measured at 24,48 and 72 or $96 \mathrm{~h}$ according to the manufacturer's instructions.

\section{Matrigel Morphogenesis Assay}

Growth factor-reduced Matrigel (BD Biosciences) was plated in 48 -well plates and incubated at $37^{\circ} \mathrm{C}$ for at least $1 \mathrm{~h}$ to allow for its gelation. IU-TAB-1cells $\left(3 \times 10^{4}\right)$ were seeded on the Matrigel, and incubated at $37^{\circ} \mathrm{C}$ overnight $(\mathrm{o} / \mathrm{n})$. Cultures were observed at $6,12,24$ and $48 \mathrm{~h}$, and photographed using a Nikon D40X camera.

\section{Thymoma Xenograft Model}

Five- to six-week-old NOD/SCID mice (obtained from the In Vivo Therapeutics Core at Indiana University Melvin and Bren Simon Cancer Center) were acclimatized for 6 days. IUTAB- 1 cells $\left(10 \times 10^{6}\right.$ cells/mouse; six mice per group $)$ mixed with Matrigel were injected subcutaneously into their flanks and allowed to set. Cells were also injected into flanks without Matrigel. Tumors were measured weekly using caliper for external measurements. Tumor volume was calculated as $L \times W^{2} / 2$, where $L$ is length and $W$ is width. All animal experiments were done under a protocol approved by the Indiana University Institutional Animal Care and Use Committee.

\section{RESULTS}

\section{IU-TAB-1 Cell Line is TE in Origin}

The primary tumor showed a pattern of type $A B$ histomorphologically composed of spindle cells admixed with variable number of lymphocytes (B-like areas; Figure 1a). To confirm the epithelial nature of the IU-TAB-1 cells, we performed 

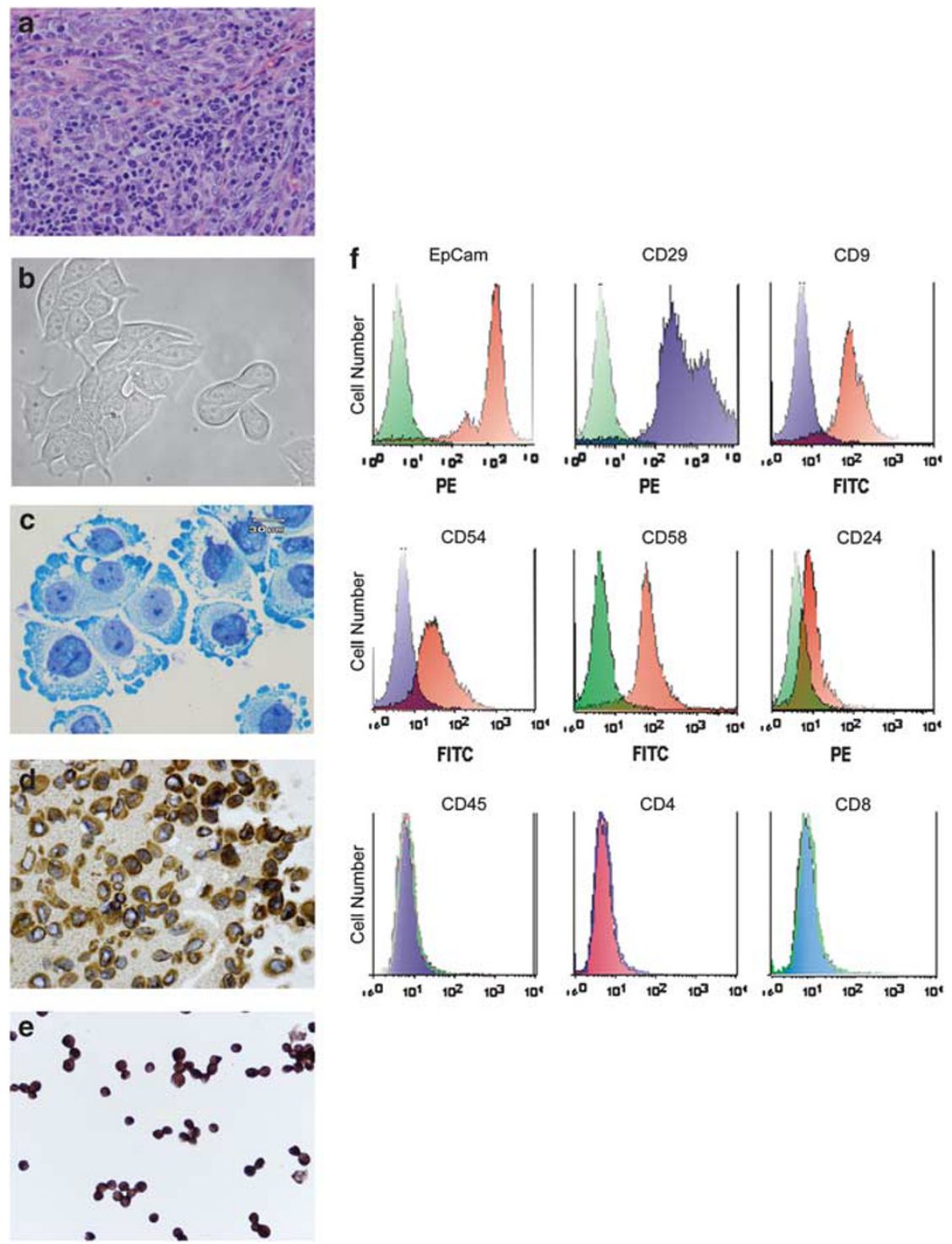

Figure 1 Morphology and origin of the IU-TAB-1 cell line are shown in (a) representative hematoxylin and eosin staining of the primary thymic tumor from which IU-TAB-1 cells were derived. (b) High-power magnification $(\times 40)$ of the cultured cell line under microscope. (c) Methylene blue staining of the cultured cell line under microscope. (d) Immunohistochemical staining with positivity for pan-cytokeratin, (high-power magnification $\times 40$ ). (e) Immunohistochemical staining with nuclear positivity for p63 (high-power magnification $\times 40$ ). (f) Flow cytometry analysis for surface expression of EpCAM, CD29, CD9, CD54, CD58, CD24, CD45, CD4 and CD8.

morphological assessment, immunohistochemical staining and fluorescence-activated cell sorting (FACS) analysis. IUTAB-1 cells grew as adherent monolayer with a cobblestone morphology characteristic of epithelial cells (Figure 1b). Methylene blue staining further verified the high nucleic acid content of the cell line with prominent nucleoli (Figure 1c). The cells were positive for pan-cytokeratin (Figure 1d), p63 (Figure 1e), and EpCAM/CD326 (Figure 1f) and negative for the lymphoid and other leukocyte markers, including CD45, and T-cell markers including CD4 and CD8 (Figure 1f). Importantly, the cells expressed CD29, CD9, CD58 and CD24 that have been shown to be common between TE cells and epidermal keratinocytes and CD54/ICAM-1 is positive only in TE cells. The rest of the markers listed in Materials and Methods were negative. These results confirm that IU-TAB-1 cell line is TE in origin. The cells, as well as the original tumor, were also negative for TdT, CD99, CD1a, c-KIT and EGFR. The tumor, but not the cell line, was focally positive for CD20 (data not shown).

\section{Most of the Chromosome Aberrations Listed in Type AB Thymomas are Present in IU-TAB-1 Cell Line}

We next assessed whether IU-TAB-1 cell line contains some of the chromosome aberrations reported earlier. Table 1 summarizes the genetic aberrations present in IU-TAB-1 in comparison with other type $\mathrm{AB}$ and histological types 
reported in the literature. Among them, aberrations in $6 \mathrm{q} 23 \mathrm{q} 25,7 \mathrm{p} 15,8 \mathrm{p}, 13 \mathrm{q}$ and $16 \mathrm{q}$ were common in all thymoma types including type $\mathrm{AB} .{ }^{21-24}$ Other aberrations listed in type $\mathrm{AB}$ thymomas include $2,3,4,5$ and 18 , which were present in IU-TAB-1 cell line. On the other hand, IU-TAB-1 cell line did not have the aberrations of 5q21-22(APC), $13 \mathrm{q} 14.3$ (RB) and 17p13.1 (p53) that were specific to a subset of more invasive type B3 thymomas. ${ }^{21,24,25}$ IU-TAB-1 exhibits some other aberrations reported in $A B$ cases such as1q, 10p, 11q21-q23,13q, 16q and 17q. These results support the relevance of IU-TAB- 1 cell line as a useful preclinical model.

\section{Relevance of IU-TAB-1 Cell Line in Comparison with the Primary Thymic Tumors}

To investigate the relevance of IU-TAB-1 cell line to the primary thymomas, a whole-genome gene expression analysis was performed on the IU-TAB- 1 cell line, and compared with 34 frozen thymomas categorized into three groups as described in Materials and Methods. Unsupervised hierarchial clustering revealed two main branches and seven subgroups (Supplementary Figure S1). All the IU-TAB-1 replicates clustered together. Of the nine $\mathrm{AB}$ tumors, six of them were in the same branch including the IU-TAB-1 patient. The remaining three $\mathrm{AB}$ samples were clustered in the second branch group.

To further assess the similarities and dissimilarities between IU-TAB- 1 cell line and its primary tumor of origin or other thymomas, we aimed for specific gene sets that may distinguish TE tumor cell line from the complex tumor microenvironment of the primary tumors (Figures 2a-d). Of the genes related to the cell surface antigens in resting and interferon- $\gamma$-treated human TE cells identified by Patel et al, ${ }^{18}$ 21 surface antigens were differentially regulated in the different groups (Figure 2a). More specifically, CD9, CD24 and CD58 were strongly upregulated in IU-TAB-1 cell line, confirming its TE nature.

To further exclude stromal origin of IU-TAB-1 cells, we compared the cell line and tumors based on their stromal content. We compared our gene expression profiles with two distinct stromal signatures reported in Webster et al. ${ }^{19}$ These signatures consist of a core set of fibroblast genes (a core set of 66 desmoid-type fibromatosis (DTF)-associated genes; DTF fibroblast signature correlated with lower tumor grade) and of a macrophage response core gene set (a core set of 112 genes-CSF1signature associated with higher tumor grade). Importantly, there was a marked downregulation of these stromal genes from both signatures in IU-TAB- 1 cells when compared with the primary tumors. This further confirms the epithelial phenotype of the cell line (Figures $2 b$ and c). As expected, differential regulation of these genes were observed in primary tumors. Interestingly, most of the group II tumors (B1-B2) clustered in the branch with high expression of these macrophage response genes, whereas group I representing $\mathrm{AB}$ tumors were in the low-expression branch.
To analyze the expression levels of the immune-systemrelated genes in IU-TAB-1 and primary tumor groups, we used the list of 199 Affymetrix ProbeSets categorized into 7 metagene clusters by Rody et al. ${ }^{20}$ The immune-systemrelated genes were mostly downregulated in IU-TAB-1 cell line, while they were differentially regulated between different groups of primary tumors (Figure 2d). Interestingly, the tumor origin of IU-TAB-1 was among the samples with very low expression of immune-system-related genes. Most of the immune-response-related genes were upregulated in group II representing B1-B2 thymomas. Taken together, these results suggest that IU-TAB-1 cell line is representative of TE cells and do not express stromal- and immune-related genes.

\section{The Functional Growth Properties of IU-TAB-1 Cell Line In Vitro and In Vivo}

To determine the in vitro growth kinetics and doubling time of IU-TAB-1 cell line, we used both trypan-blue dye exclusion method and BrdU cell proliferation assay (Figures $3 a$ and $b$ ). The doubling time was calculated as $48 \mathrm{~h}$ (Figure 3a). The morphogenic properties of IU-TAB- 1 cells were evaluated by analyzing these features in Matrigel cultures. After incubation for $12 \mathrm{~h}$, active cell locomotion was observed in Matrigel, with the formation of luminal-like clusters, and with some expansion of the clusters over time (Figure 3c). These clusters resembled the structures formed in Matrigel by luminal MCF7 or ZR75-1 breast cancer cells, and were distinct for the cellular networks formed by more invasive mesenchymal-like breast cancer cells (as MDA-MB-231 cell line).

To assess whether IU-TAB-1 cells were capable of forming tumors in immunodeficient NOD/SCID mice, cells were implanted subcutaneously admixed with Matrigel or alone. As shown in Figure 3d, IU-TAB-1 cells are tumorigenic, forming ectopic tumors that reached a volume of $1000 \mathrm{~mm}^{3}$ at around 130 days when in Matrigel-plugs, and around 148 days when implanted alone. Importantly, the kinetics of tumor development is consistent with the indolent nature of $\mathrm{AB}$ thymomas. The mice were killed and paraffin-embedded sections were prepared to stain with $\mathrm{H} \& \mathrm{E}$ and pan-cytokeratin as an epithelial cell marker. The malignant epithelial nature of xenograft IU-TAB-1 tumor was confirmed histologically (H\&E; Figure 3e) and immunohistochemically (pan-cytokeratin and p63) (Figures $3 \mathrm{f}$ and $\mathrm{g}$ ). This demonstrates that IU-TAB-1 line contains cells with tumor-initiating properties.

\section{DISCUSSION}

Preclinical models have been important tools to understand the molecular pathology of human cancers and to develop better therapeutics. Various human cancer cell lines isolated from patient tumors have provided extensive information for the cellular and molecular mechanisms of cancer biology. For example, the development of multiple breast cancer cell lines and xenograft models contributed to our understanding of the molecular targets in breast cancer tumorigenesis and 

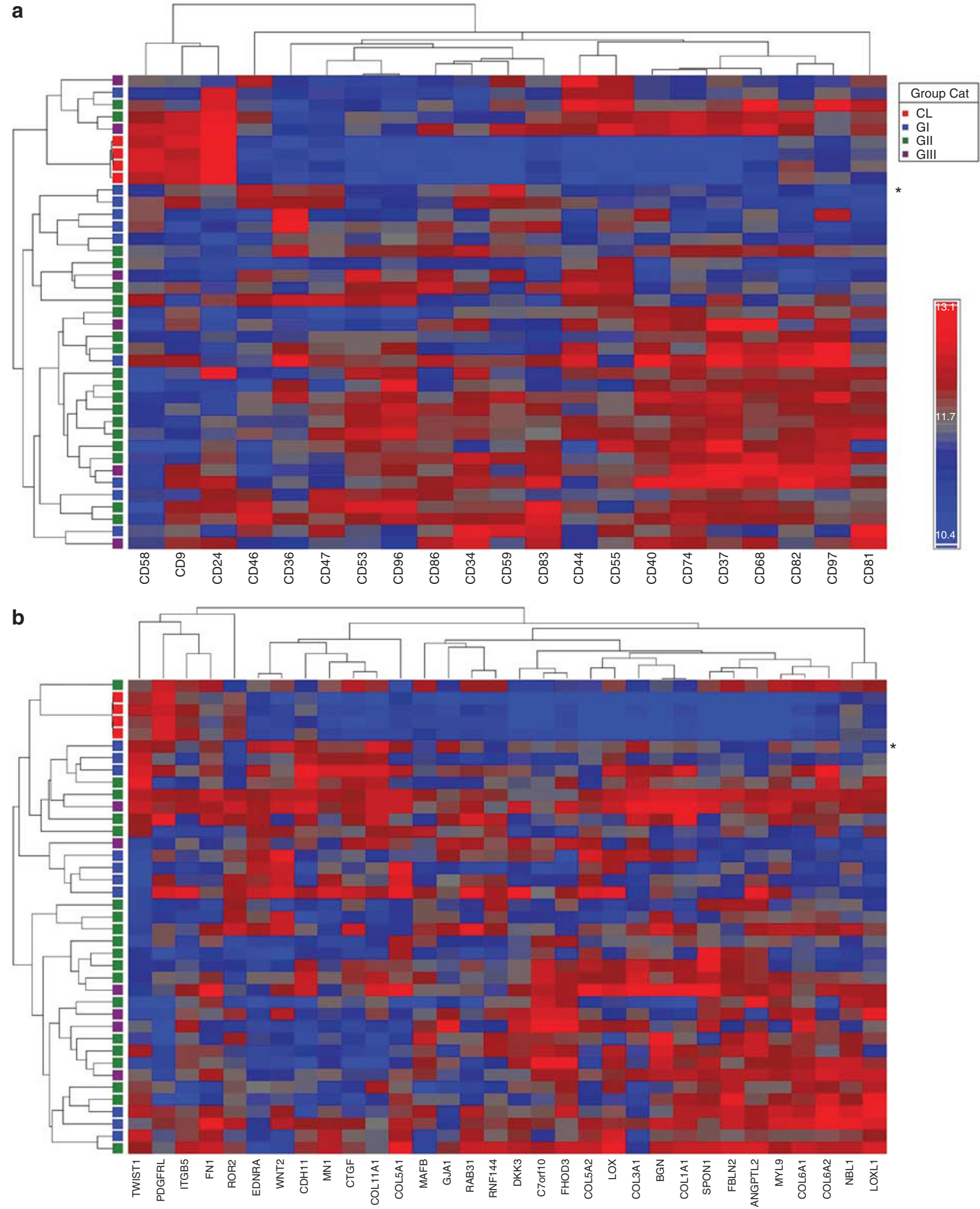

Figure 2 Selected gene sets of differentially regulated genes between IU-TAB-1 cell line and 34 frozen thymomas. (a) TE cell surface antigens. (b) Stroma-related gene signature, desmoid-type fibromatosis (DTF)-associated genes DTF fibroblast signature. (c) Stroma-related gene signature, a macrophage response core gene set-CSF1signature. (d) Immune-system-related genes. CL =Cell line, group I (type AB), group II (types B1-B2) and group III (type B3).*Represents the primary tumor where IU-TAB-1 cell line was derived. One duplicate for group 1 was also included for cluster analysis. 

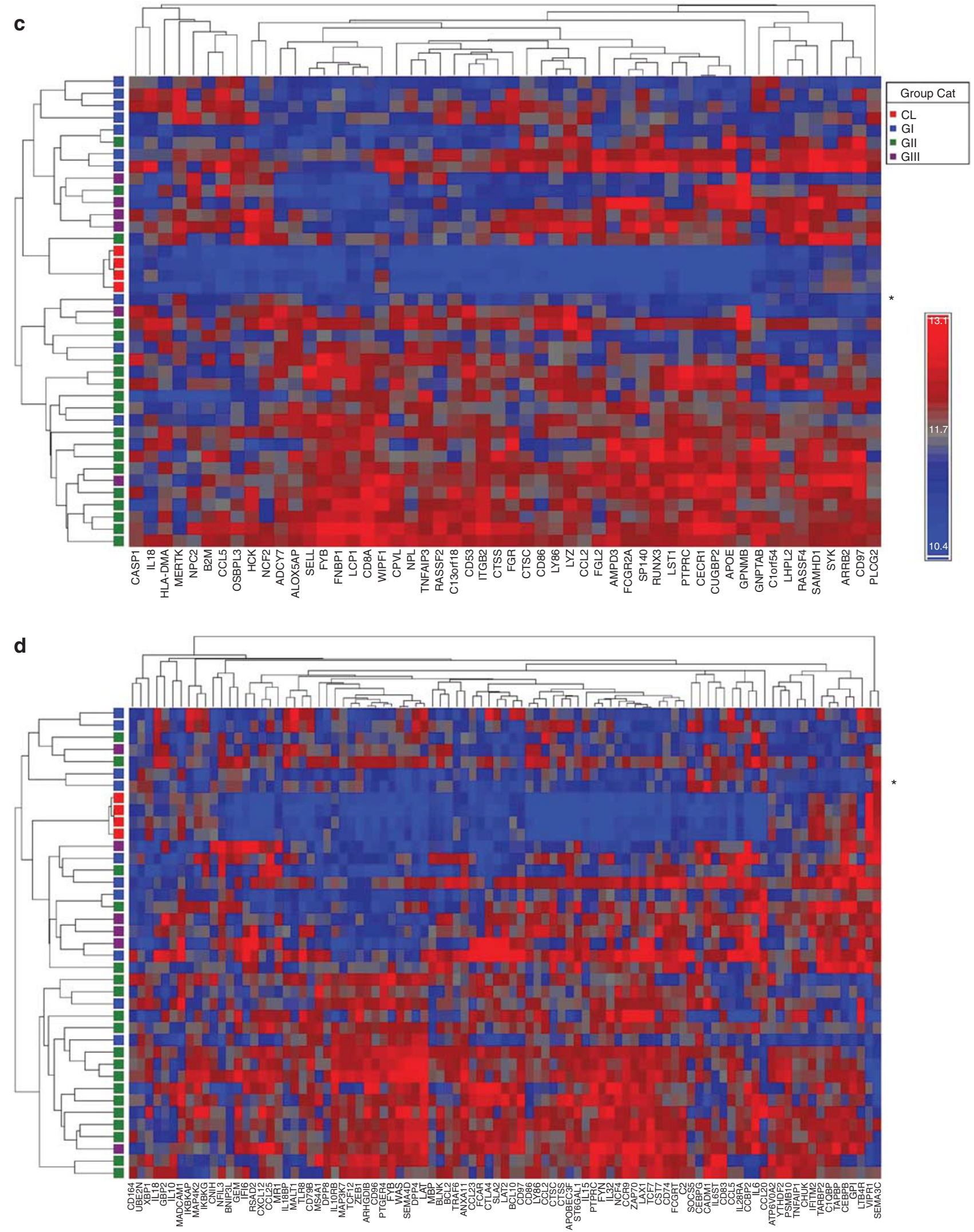

Figure 2 Continued. 

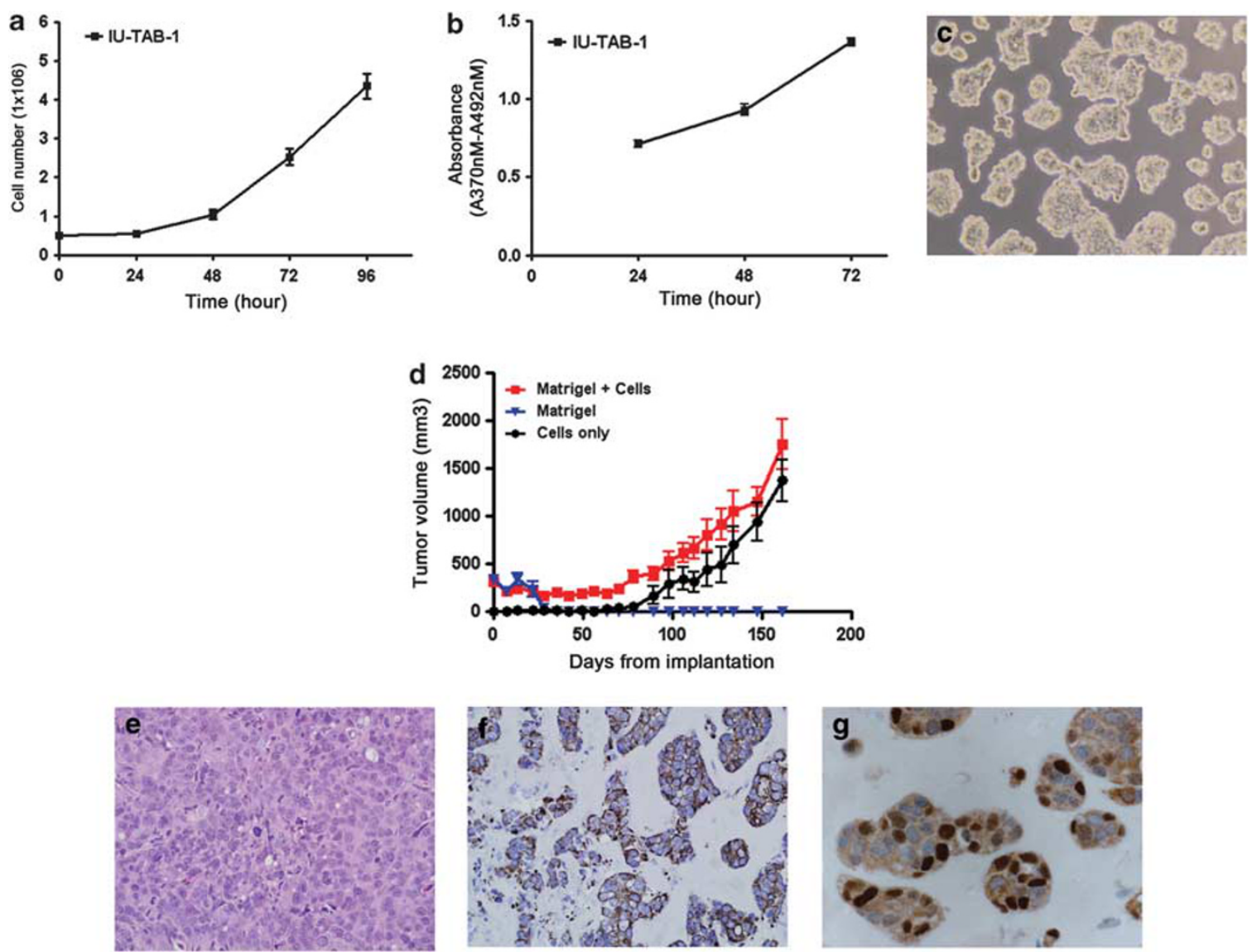

Figure 3 The in vitro and in vivo functional properties of IU-TAB-1. The growth curve of IU-TAB-1 cell line was shown using (a) trypan-blue dye exclusion method and (b) BrdU cell proliferation colorimetric ELISA kit (Roche Diagnostics). Data points represent the means of three independent experiments. (c) Morphogenesis assay of IU-TAB-1 in Matrigel. (d) The tumorigenic potential of IU-TAB-1 cells was assessed by implanting IU-TAB-1 cells into the flanks of NOD/SCID mice with cells alone or mixed with low growth factor-reduced Matrigel (Becton Dickinson, Bedford, MA, USA). (e and $\mathbf{f}$ ) Represent the staining of the tumors derived from IU-TAB-1 cells implanted into NOD/SCID mouse. (e) Hematoxylin and eosin and (f) immunohistochemical staining with positivity for pan-cytokeratin, (high-power magnification $\times 40$ ). (g) Immunohistochemical staining with nuclear positivity for p63 (high-power magnification $\times 40$ ).

introduced novel targeted therapies in the clinic. ${ }^{26}$ However, to date, this has not been the case for thymic malignancies. Mechanistic information regarding the tumorigenesis, progression and recurrence of thymic neoplasms is extremely limited due to the lack of relevant preclinical models. To date, only a few cell lines from tumors of the thymus have been established representing mostly thymic carcinoma. ${ }^{13-15}$ In this study, we report a detailed characterization of a novel thymoma cell line (IU-TAB-1), which was derived from a patient with the stage II thymoma (WHO-type $\mathrm{AB}$ tumor) and its molecular comparison in regard to the primary thymomas.

Histological and immunophenotype and FACS analyses confirmed the TE nature of this cell line by the presence of epithelial cell markers (pan-cytokeratin and EpCAM/ CD326), the presence of cell surface markers of TE cells ${ }^{18}$ and nuclear $\mathrm{p} 63,{ }^{27}$ and by the absence of lymphoid and other leukocyte markers as well as T-cell markers. ${ }^{28-30}$ We verified that IU-TAB-1 cell line does not express c-KIT (CD117) and
EGFR markers, which are typically expressed in thymic carcinomas. $^{31,32}$

Genetic alterations in thymomas have been correlated with the histological WHO subtype and the clinical behavior. ${ }^{10,21-25,33,34}$ Using conventional cytogenetics analysis, we identified and correlated chromosomal alterations in the IU-TAB-1 cell line with the reported aberrations. At first glance, there were a surprising large number of aberrations that were identified in IU-TAB-1. This data must be viewed in comparison with data from other benign and malignant cell lines. High frequency of chromosomal aberrations has been detected in cell lines derived from other organ sites. Cell lines derived from 'normal' tissue often show significant number of chromosomal aberrations. For example, MCF10A cell line, a commonly 'normal' breast cell line, harbors multiple chromosome aberrations, some of which might contribute to its immortalized phenotype. ${ }^{35}$ In addition, even benign lesions such as fibroadenomas or colonic adenomas harbor chromosomal aberrations, ${ }^{36,37}$ albeit at a lower 
frequency that malignant tumors. Cytogenetic studies revealed recurrent chromosome alterations in colorectal adenomas. ${ }^{37}$ Cell lines established from colorectal adenomas harbor various chromosome aberrations, ${ }^{38,39}$ suggesting the involvement of these alterations in the transformation to malignant phenotype. Furthermore, we among others have documented that thymomas are tumors that can behave in a malignant manner. Abnormalites identified in tumors have been attributed to the heterogeneous nature of primary tumors, with the aberrant subset population surviving in the culture system environment. Neve et $\mathrm{l}^{26}$ has reported that the breast cancer cell lines carry more aberrations than primary tumors, although they retained many biological and genomic properties of primary tumors. These studies also support the hypothesis that IU-TAB-1 cell line could provide important knowledge as experimental models for understanding the thymoma biology and testing new drugs.

Our data are consistent with phenotypes described in type $\mathrm{AB}$ thymomas reported in earlier studies. ${ }^{10,21-23,33,34}$ Chromosome 6q abnormalities, as noted in IU-TAB-1, are among the most frequently seen in thymic neoplasms. Inoue et $a l^{21}$ has reported that all the histological types except $\mathrm{A}$ showed multiple recurrent allelic imbalances at chromosome loci besides chromosome 6 aberrations. In addition, our studies showed that all histological types, albeit with different frequencies, can invade and metastasize to extrathoracic sites. ${ }^{5,6}$ Also of note, aberrations in chromosome 20 p13 $(\operatorname{add}(20)(\mathrm{p} 13)$ were observed in IU-TAB-1. Thymoma insertional mutation-1 transgenic mice, which carry a transgene insertional mutagenesis syntenic to human chromosome 20p13, have been shown to develop thymomas. ${ }^{40}$

Using gene expression profiling based approach, we further analyzed the similarities between the cell line and primary thymomas. IU-TAB-1 retained some of the genes, but clustered separately from primary tumors. These observations can be at least partially explained on the basis of the following: Prior studies on other tumors have also observed that cell lines and primary tumors cluster differently; ${ }^{41}$ The absence of tumor microenvironment in the cell line is one of the obvious reasons for the cell line to cluster differently. In this regard, we show that IU-TAB-1 lacks both stromal and immune-system-related genes in contrast to the primary tumor groups, confirming the differences due to the multicellular nature of primary tumors.

The ability of human cancer cell lines to form tumors in immuno-compromised mouse models allows analysis of in vivo drug efficacy as preclinical proof-of- concept validation. We have shown that IU-TAB-1 is tumorigenic in vivo underlining its potential as a preclinical model for thymic neoplasms. Of note, the slow kinetics of tumor development in the xenograft model of IU-TAB-1 is consistent with its origin in thymoma type $\mathrm{AB}$ and goes hand in hand with the features seen in $\mathrm{Ma}-$ trigel morphogenesis assay. These combinatorial results make IU-TAB-1 cell line relevant in the investigation of thymomas.
In conclusion, we report a novel preclinical model for the study of human thymoma that should provide an excellent tool to study the relevance and functional role of novel therapeutic targets in thymomas.

Supplementary Informationaccompanies the paper on the Laboratory Investigation website (http://www.laboratoryinvestigation.org)

\section{ACKNOWLEDGEMENTS}

This work was supported in part by the Barbara P Hochberg Foundation and William P Loehrer Family Research Fund.

\section{DISCLOSURE/CONFLICT OF INTEREST}

The authors declare no conflict of interest.

1. Rosai J, Sobin LH. Histological typing of tumors. In: World Health Organization International Histological Classification of Tumours, Heidelberg, 1999.

2. Travis WD. World Health Organization, International Agency for Research on Cancer, International Association for the Study of Lung Cancer, International Academy of Pathology. Pathology and genetics of tumours of the lung, pleura, thymus and heart; Lyon, Oxford: IARC Press. Oxford University Press (distributor), 2004, p 344.

3. Masaoka A, Monden Y, Nakahara K, et al. Follow-up study of thymomas with special reference to their clinical stages. Cancer 1981; 48:2485-2492.

4. Casey EM, Kiel PJ, Loehrer Sr PJ. Clinical management of thymoma patients. Hematol Oncol Clin North Am 2008;22:457-473.

5. Jain RK, Mehta RJ, Henley JD, et al. WHO types A and AB thymomas: not always benign. Mod Pathol 2010;23:1641-1649.

6. Vladislav T, Jain RK, Alvarez R, et al. Extrathoracic metastases of thymic origin: a review of 35 cases. Mod Pathol 2012;25:370-377.

7. Loehrer Sr PJ, Jiroutek M, Aisner S, et al. Combined etoposide, ifosfamide, and cisplatin in the treatment of patients with advanced thymoma and thymic carcinoma: an intergroup trial. Cancer 2001;91:2010-2015.

8. Hanna N, Gharpure VS, Abonour R, et al. High-dose carboplatin with etoposide in patients with recurrent thymoma: the Indiana University experience. Bone Marrow Transplant 2001;28:435-438.

9. Okereke IC, Kesler KA, Morad MH, et al. Prognostic indicators after surgery for thymoma. Ann Thorac Surg 2010;89:1071-1077.

10. Girard N, Shen R, Guo T, et al. Comprehensive genomic analysis reveals clinically relevant molecular distinctions between thymic carcinomas and thymomas. Clin Cancer Res 2009;15:6790-6799.

11. Giaccone G. Treatment of malignant thymoma. Curr Opin Oncol 2005;17:140-146.

12. Giaccone $G$, Rajan A, Ruijter R, et al. Imatinib mesylate in patients with WHO B3 thymomas and thymic carcinomas. J Thorac Oncol 2009;4:1270-1273.

13. Kuzume T, Kubonishi I, Takeuchi S, et al. Establishment and characterization of a thymic carcinoma cell line (Ty-82) carrying $t(15 ; 19)$ (q15;p13) chromosome abnormality. Int J Cancer 1992;50:259-264.

14. Ehemann V, Kern MA, Breinig M, et al. Establishment, characterization and drug sensitivity testing in primary cultures of human thymoma and thymic carcinoma. Int J Cancer 2008;122:2719-2725.

15. Inai $\mathrm{K}$, Takagi $\mathrm{K}$, Takimoto $\mathrm{N}$, et al. Multiple inflammatory cytokineproductive ThyL-6 cell line established from a patient with thymic carcinoma. Cancer Sci 2008;99:1778-1784.

16. Langdon SP (ed). Cancer Cell Culture: Methods and Protocols: Springer Protocols, 2003.

17. Badve S, Goswami C, Gökmen-Polar Y, et al. Molecular analysis of thymoma. PLoS ONE 2012;7:e42669.

18. Patel DD, Whichard LP, Radcliff G, et al. Characterization of human thymic epithelial cell surface antigens: phenotypic similarity of thymic epithelial cells to epidermal keratinocytes. J Clin Immunol 1995; 15:80-92.

19. Webster JA, Beck AH, Sharma M, et al. Variations in stromal signatures in breast and colorectal cancer metastases. J Pathol 2010;222:158-165. 
20. Rody A, Holtrich U, Pusztai $\mathrm{L}$, et al. T-cell metagene predicts a favorable prognosis in estrogen receptor-negative and HER2-positive breast cancers. Breast Cancer Res 2009;11:R15.

21. Inoue M, Starostik P, Zettl A, et al. Correlating genetic aberrations with World Health Organization-defined histology and stage across the spectrum of thymomas. Cancer Res 2003;63:3708-3715.

22. Penzel R, Hoegel J, Schmitz W, et al. Clusters of chromosomal imbalances in thymic epithelial tumours are associated with the WHO classification and the staging system according to Masaoka. Int J Cancer 2003;105:494-498.

23. Lee $\mathrm{GY}$, Yang $\mathrm{Wl}$, Jeung $\mathrm{HC}$, et al. Genome-wide genetic aberrations of thymoma using CDNA microarray based comparative genomic hybridization. BMC Genomics 2007;8:305.

24. Zettl $A$, Strobel $P$, Wagner $K$, et al. Recurrent genetic aberrations in thymoma and thymic carcinoma. Am J Pathol 2000;157:257-266.

25. Strobel P, Marx A, Zettl A, et al. Thymoma and thymic carcinoma: an update of the WHO Classification 2004. Surg Today 2005;35: 805-811.

26. Neve RM, Chin K, Fridlyand J, et al. A collection of breast cancer cell lines for the study of functionally distinct cancer subtypes. Cancer Cell 2006;10:515-527.

27. Dotto J, Pelosi G, Rosai J. Expression of p63 in thymomas and normal thymus. Am J Surg Pathol 20071161-1167.

28. Berezowski K, Grimes MM, Gal A, et al. CD5 immunoreactivity of epithelial cells in thymic carcinoma and CASTLE using paraffinembedded tissue. Am J Clin Pathol 1996;106:483-486.

29. Hishima T, Fukayama M, Fujisawa $M$, et al. CD5 expression in thymic carcinoma. Am J Pathol 1994;145:268-275.

30. Kornstein MJ, Rosai J. CD5 labeling of thymic carcinomas and other nonlymphoid neoplasms. Am J Clin Pathol 1998;109:722-726.
31. Henley JD, Cummings OW, Loehrer Sr PJ. Tyrosine kinase receptor expression in thymomas. J Cancer Res Clin Oncol 2004;130: 222-224.

32. Henley JD, Koukoulis GK, Loehrer Sr PJ. Epidermal growth factor receptor expression in invasive thymoma. J Cancer Res Clin Oncol 2002;128:167-170.

33. Dal Cin P, De Wolf-Peeters C, Aly MS, et al. Ring chromosome 6 as the only change in a thymoma. Genes Chromosomes Cancer 1993;6: 243-244.

34. Goh SG, Lau LC, Sivaswaren C, et al. Pseudodicentric (16;12)(q11;p11.2) in a type $A B$ (mixed) thymoma. Cancer Genet Cytogenet 2001;131: 42-47.

35. Cowell JK, LaDuca J, Rossi MR, et al. Molecular characterization of the $\mathrm{t}(3 ; 9)$ associated with immortalization in the MCF10A cell line. Cancer Genet Cytogenet 2005;163:23-29.

36. Rizou H, Bardi G, Arnaourti $M$, et al. Metaphase and interphase cytogenetics in fibroadenomas of the breast. In Vivo 2004;18:703-711.

37. Muleris M, Zafrani B, Validire $P$, et al. Cytogenetic study of 30 colorectal adenomas. Cancer Genet Cytogenet 1994;74:104-108.

38. Willson JK, Bittner GN, Oberley TD, et al. Cell culture of human colon adenomas and carcinomas. Cancer Res 1987;47:2704-2713.

39. Paraskeva C, Finerty S, Mountford RA, et al. Specific cytogenetic abnormalities in two new human colorectal adenoma-derived epithelial cell lines. Cancer Res 1989;49:1282-1286.

40. Nakajima $\mathrm{H}$, Nakajima $\mathrm{HO}$, Soonpaa $\mathrm{MH}$, et al. Heritable lymphoepithelial thymoma resulting from a transgene insertional mutation. Oncogene 2000;19:32-38.

41. Ross DT, Perou CM. A comparison of gene expression signatures from breast tumors and breast tissue derived cell lines. Dis Markers 2001;17:99-109. 